\title{
Discrete quantum Boltzmann equation
}

Cite as: AIP Conference Proceedings 2132, 130011 (2019); https://doi.org/10.1063/1.5119631

Published Online: 05 August 2019

Niclas Bernhoff

\section{ARTICLES YOU MAY BE INTERESTED IN}

Shocks in the light of discrete velocity models

AIP Conference Proceedings 2132, 060002 (2019); https://doi.org/10.1063/1.5119542

A kinetic model of reactive crystal surfaces

AIP Conference Proceedings 2132, 130003 (2019); https://doi.org/10.1063/1.5119623

Theoretical modeling of vibrational nonequilibrium flows behind strong shock waves

AIP Conference Proceedings 2132, 130009 (2019); https://doi.org/10.1063/1.5119629

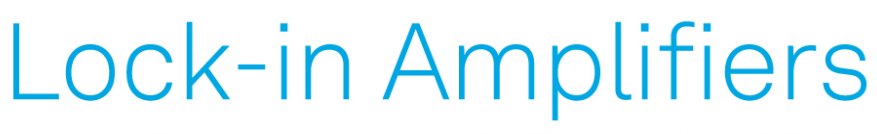

... and more, from DC to $600 \mathrm{MHz}$

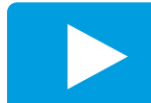

Watch 


\title{
Discrete Quantum Boltzmann Equation
}

\author{
Niclas Bernhoff \\ Department of Mathematics and Computer Science, Karlstad University, 65188 Karlstad, Sweden \\ Corresponding author: niclas.bernhoff@kau.se
}

\begin{abstract}
In this work, we consider a Boltzmann equation for anyons. In particular, we study a general discrete velocity model of the equation, where the velocity variable is assumed to only take values from a given finite - such that the (finite) number of velocities is arbitrary - set of velocities. Included, as two limiting cases, is the discrete quantum Boltzmann equation (NordheimBoltzmann/Uehling-Uhlenbeck equation) for bosons and fermions. Mass, momentum, and energy are assumed to be conserved during collisions, and considering suitable discrete velocity models, they will also be the only collision invariants. The equilibrium distributions will be given by a transcendental equation, and only in some few cases - including the two limiting cases where they are Planckians - they will be explicitly expressed. However, there is an H-theorem, and therefore one can prove that for the spatially homogeneous equation, as time tends to infinity, as well as, for the steady equation in a half-space with slab-symmetry, as the space variable tends to infinity, the distribution function converges to an equilibrium distribution. Linearizing around an equilibrium distribution in a suitable way, we find that the obtained linearized operator has similar properties as the corresponding linearized operator for the discrete Boltzmann equation: e.g. it is symmetric and positive semi-definite. Hence, previously obtained results for the spatially homogeneous Cauchy problem and the steady half-space problem in a slab symmetry for the discrete Boltzmann equation, can be applied also in the considered quantum case.
\end{abstract}

\section{INTRODUCTION}

In quantum mechanics the elementary particles, quantum particles, are either bosons or fermions, if one consider a space of three (or more) dimensions. However, in a space of dimension two (or one), there are also other possibilities, as was first noted by Leinaas and Myrheim [1]. Those latter quantum particles, obeying a fractional statistics, were by Wilczek [2] called "anyons". In 1928 Nordheim presented the Nordheim-Boltzmann equation [3], a semi-classical quantum Boltzmann equation for bosons and fermions, also known as the Uehling-Uhlenbeck equation [4] in literature. In 1995, Bhaduri, Bhalerao, and Murthy generalized the Nordheim-Boltzmann equation for bosons and fermions, to yield also for particles obeying Haldane statistics [5], or fractional exclusion statistics, by a suitable modification [6]. For some mathematical studies of this equation, see e.g. [7, 8, 9]. In this paper we present a general discrete velocity model (DVM) of Boltzmann equation for anyons - or Haldane statistics - and derive some properties for it concerning: equilibrium distributions, $\mathcal{H}$-theorem(s), trend to equilibrium for spatially homogeneous and planar stationary systems, respectively, linearized collision operator, Cauchy problem for the spatially homogeneous equation, and steady half-space problems in a slab-symmetry. The approach is similar to the one for the "classical" discrete Boltzmann equation $[10,11,12]$.

\section{DISCRETE BOLTZMANN EQUATION FOR HALDANE STATISTICS}

The discrete Boltzmann equation for anyons, or rather particles obeying Haldane statistics, reads

$$
\frac{\partial F_{i}}{\partial t}+\mathbf{p}_{i} \cdot \nabla_{\mathbf{x}} F_{i}=Q_{i}^{\alpha}(F), i=1, \ldots, N, 0<\alpha<1,
$$

where $\mathcal{P}=\left\{\mathbf{p}_{1}, \ldots, \mathbf{p}_{N}\right\} \subset \mathbb{R}^{d}$ - with $d=1$ or $d=2$ in applications - is a finite set $(N$ is an arbitrary positive integer) and $F=\left(F_{1}, \ldots, F_{N}\right)$, with $F_{i}=F_{i}(\mathbf{x}, t)=F\left(\mathbf{x}, t, \mathbf{p}_{i}\right)$ and $0 \leq F_{i} \leq \frac{1}{\alpha}$, is the distribution function. We will also consider the limiting cases $\alpha=0$ (without any upper bound on $F_{i}$ ), corresponding to the discrete Nordheim-Boltzmann equation

31st International Symposium on Rarefied Gas Dynamics

AIP Conf. Proc. 2132, 130011-1-130011-9; https://doi.org/10.1063/1.5119631

Published by AIP Publishing. 978-0-7354-1874-5/\$30.00 
for bosons, and $\alpha=1$, corresponding to the discrete Nordheim-Boltzmann equation for fermions. Unlike for the intermediate cases, in the limiting cases for bosons and fermions, the three dimensional case $d=3$ is highly relevant in applications. Below we will, for brevity, make the technical assumption that $0<F_{i}<\frac{1}{\alpha}$ for $i=1, \ldots, N$, if necessary, without stating it explicitly.

Remark 1 For a function $g=g(\mathbf{p})$ (possibly depending on more variables than $\mathbf{p}$ ), we will identify $g$ with its restrictions to the points $\mathbf{p} \in \mathcal{P}$, i.e.

$$
g=\left(g_{1}, \ldots, g_{N}\right), g_{i}=g\left(\mathbf{p}_{i}\right), i=1, \ldots, N .
$$

The collision operators $Q_{i}^{\alpha}(F), i=1, \ldots, N$, in the system (1) are given by

$$
\begin{aligned}
Q_{i}^{\alpha}(F) & =\sum_{j, k, l=1}^{N} \Gamma_{i j}^{k l}\left(F_{k} F_{l} \Psi_{\alpha}\left(F_{i}\right) \Psi_{\alpha}\left(F_{j}\right)-F_{i} F_{j} \Psi_{\alpha}\left(F_{k}\right) \Psi_{\alpha}\left(F_{l}\right)\right) \\
& =\sum_{j, k, l=1}^{N} \Gamma_{i j}^{k l} \Psi_{\alpha}\left(F_{i}\right) \Psi_{\alpha}\left(F_{j}\right) \Psi_{\alpha}\left(F_{k}\right) \Psi_{\alpha}\left(F_{l}\right)\left(\frac{F_{k}}{\Psi_{\alpha}\left(F_{k}\right)} \frac{F_{l}}{\Psi_{\alpha}\left(F_{l}\right)}-\frac{F_{i}}{\Psi_{\alpha}\left(F_{i}\right)} \frac{F_{j}}{\Psi_{\alpha}\left(F_{j}\right)}\right), \\
\Psi_{\alpha}\left(F_{i}\right) & =\left(1-\alpha F_{i}\right)^{\alpha}\left(1+(1-\alpha) F_{i}\right)^{1-\alpha}
\end{aligned}
$$

where it is assumed that the collision coefficients $\Gamma_{i j}^{k l}, 1 \leq i, j, k, l \leq N$, satisfy the relations

$$
\Gamma_{i j}^{k l}=\Gamma_{j i}^{k l}=\Gamma_{k l}^{i j} \geq 0
$$

with equality unless the conservation laws

$$
\mathbf{p}_{i}+\mathbf{p}_{j}=\mathbf{p}_{k}+\mathbf{p}_{l} \text { and }\left|\mathbf{p}_{i}\right|^{2}+\left|\mathbf{p}_{j}\right|^{2}=\left|\mathbf{p}_{k}\right|^{2}+\left|\mathbf{p}_{l}\right|^{2}
$$

are satisfied. For bosons $(\alpha=0)$ and fermions $(\alpha=1)$, we have the classical factors

$$
\Psi_{0}\left(F_{i}\right)=1+F_{i} \text { and } \Psi_{1}\left(F_{i}\right)=1-F_{i},
$$

respectively, and e.g. for $\alpha=1 / 2$ (semions) we have the factor

$$
\Psi_{1 / 2}\left(F_{i}\right)=\sqrt{1-\frac{F_{i}^{2}}{4}} .
$$

One can easily show that, due to the relations (3), we have that

$$
\begin{aligned}
\left\langle H, Q^{\alpha}(F)\right\rangle=\frac{1}{4} \sum_{i, j, k, l=1}^{N} \Gamma_{i j}^{k l} \Psi_{\alpha}\left(F_{i}\right) \Psi_{\alpha}\left(F_{j}\right) \Psi_{\alpha}\left(F_{k}\right) \Psi_{\alpha}\left(F_{l}\right)\left(H_{i}+H_{j}-H_{k}-H_{l}\right) & \left(\frac{F_{k}}{\Psi_{\alpha}\left(F_{k}\right)} \frac{F_{l}}{\Psi_{\alpha}\left(F_{l}\right)}-\frac{F_{i}}{\Psi_{\alpha}\left(F_{i}\right)} \frac{F_{j}}{\Psi_{\alpha}\left(F_{j}\right)}\right),
\end{aligned}
$$

where $\langle\cdot, \cdot\rangle$ - here and below - denotes the standard scalar product on $\mathbb{R}^{N}$. Then, by substituting $H=\log \frac{F}{\Psi_{\alpha}(F)}$ in equality (5), we obtain that

$$
\begin{array}{r}
\left\langle\log \frac{F}{\Psi_{\alpha}(F)}, Q^{\alpha}(F)\right\rangle=\frac{1}{4} \sum_{i, j, k=1}^{N} \Gamma_{i j}^{k l} \Psi_{\alpha}\left(F_{i}\right) \Psi_{\alpha}\left(F_{j}\right) \Psi_{\alpha}\left(F_{k}\right) \Psi_{\alpha}\left(F_{l}\right)\left(\log \left(\frac{F_{i}}{\Psi_{\alpha}\left(F_{i}\right)} \frac{F_{j}}{\Psi_{\alpha}\left(F_{j}\right)}\right)-\log \left(\frac{F_{k}}{\Psi_{\alpha}\left(F_{k}\right)} \frac{F_{l}}{\Psi_{\alpha}\left(F_{l}\right)}\right)\right) \\
\left(\frac{F_{k}}{\Psi_{\alpha}\left(F_{k}\right)} \frac{F_{l}}{\Psi_{\alpha}\left(F_{l}\right)}-\frac{F_{i}}{\Psi_{\alpha}\left(F_{i}\right)} \frac{F_{j}}{\Psi_{\alpha}\left(F_{j}\right)}\right) \leq 0 . \quad(6)
\end{array}
$$


The inequality (6) is obtained by using the relation

$$
(z-y) \log \frac{y}{z} \leq 0
$$

with equality if and only if $y=z$, which is valid for all $y, z \in \mathbb{R}_{+}$. Hence, we have equality in the inequality (6) if and only if

$$
\frac{F_{i}}{\Psi_{\alpha}\left(F_{i}\right)} \frac{F_{j}}{\Psi_{\alpha}\left(F_{j}\right)}=\frac{F_{k}}{\Psi_{\alpha}\left(F_{k}\right)} \frac{F_{l}}{\Psi_{\alpha}\left(F_{l}\right)},
$$

for all indices such that $\Gamma_{i j}^{k l} \neq 0$.

A collision invariant is a function $\phi=\phi(\mathbf{p})$, such that

$$
\phi_{i}+\phi_{j}=\phi_{k}+\phi_{l}
$$

for all indices $1 \leq i, j, k, l \leq N$, such that $\Gamma_{i j}^{k l} \neq 0$. The collision invariants are assumed to be of the form

$$
\phi=a+\mathbf{b} \cdot \mathbf{p}+c|\mathbf{p}|^{2}
$$

for some $a, c \in \mathbb{R}$ and $\mathbf{b} \in \mathbb{R}^{d}$. In general, DVMs can have other, so called spurious (or nonphysical), collision invariants than the (physical) collision invariants (9). DVMs without spurious collision invariants are called normal and methods of their construction are described in e.g. $[13,14,15]$. Our restriction to normal models is not necessary in our general reasoning, but is motivated by the desire to have the same number of collision invariants as in the continuous case. For normal DVMs the equation

$$
\left\langle Q^{\alpha}(F), \phi\right\rangle=0
$$

has the general solution (9).

A Maxwellian distribution (or just a Maxwellian) is a function $M=M(\xi)$, such that (for normal DVMs)

$$
M=e^{\phi}=K e^{\mathbf{b} \cdot \xi+c|\xi|^{2}}, K=e^{a}>0
$$

where $\phi$ is a collision invariant. There is equality in inequality (6), if and only if $\log \frac{F}{\Psi_{\alpha}(F)}$ is a collision invariant (take the logarithm of equality (7)), or equivalently, if and only if $\frac{F}{\Psi_{\alpha}(F)}$ is a Maxwellian $M$, i.e. the equilibrium distributions $P$ are given by the transcendental equation, cf. [16] for the continuous case,

$$
\frac{P}{\Psi_{\alpha}(P)}=M
$$

Note that, by solving equation (11), for bosons and fermions, one obtain the Planckians

$$
P=\frac{M}{1-M} \text { and } P=\frac{M}{1+M}
$$

respectively (observe that for bosons there is the restriction $M_{i}<1, i=1, \ldots, N$, on the Maxwellians, since $\frac{P_{i}}{1+P_{i}}<1$ ), and for semions $(\alpha=1 / 2)$ the equilibrium distribution

$$
P=\frac{1}{\sqrt{\frac{1}{4}+M^{-2}}} .
$$




\section{$\mathcal{H}$-THEOREM AND TREND TO EQUILIBRIUM}

We define an $\mathcal{H}$-function

$$
\mathcal{H}[F]=\mathcal{H}[F](x)=\sum_{i=1}^{N} p_{i}^{1} \mu\left(F_{i}(x)\right)
$$

where, cf. [8],

$$
\mu(y)=\left\{\begin{array}{l}
y \log y+(1-\alpha y) \log (1-\alpha y)-(1+(1-\alpha) y) \log (1+(1-\alpha) y) \text { if } 0<y<\frac{1}{\alpha} . \\
0 \text { if } y=0 \text { or }(\text { if } \alpha \neq 0) y=\frac{1}{\alpha}
\end{array} .\right.
$$

For the planar stationary system

$$
B \frac{d F}{d x}=Q^{\alpha}(F), \text { with } B=\operatorname{diag}\left(p_{1}^{1}, \ldots, p_{N}^{1}\right), x \in \mathbb{R}_{+},
$$

we obtain an $\mathcal{H}$-theorem

$$
\frac{d}{d x} \mathcal{H}[F]=\sum_{i=1}^{N} p_{i}^{1} \frac{d F_{i}}{d x} \log \frac{F_{i}}{\Psi_{\alpha}\left(F_{i}\right)}=\left\langle\log \frac{F}{\Psi_{\alpha}(F)}, Q^{\alpha}(F)\right\rangle \leq 0,
$$

with equality if, and only if, $F$ is an equilibrium distribution. We introduce the moments

$$
\left\{\begin{array}{l}
j_{1}=\langle B \mathbf{1}, F\rangle \\
j_{i+1}=\left\langle B p^{i}, F\right\rangle, i=1, \ldots, d, \mathbf{1}=(1, \ldots, 1) \in \mathbb{R}^{N} \\
j_{d+2}=\left\langle B|\mathbf{p}|^{2}, F\right\rangle
\end{array}\right.
$$

By applying relation (10) for the system (13), one obtain that $j_{1}, \ldots, j_{d+2}$ are independent of $x$ in the planar stationary case. For some fixed $j_{1}, \ldots, j_{d+2}$, we denote by $\mathbb{P}$ the manifold of all equilibrium distributions $F=P$ (given by equation (11)) with the moments (14). Then one can show the following theorem by arguments similar to the ones used for the discrete Boltzmann equation in [17] (or, also [18]).

Theorem 1 If $F=F(x)$ is a solution to the system (13), such that $0 \leq F_{i} \leq \frac{1}{\alpha}$ ( $F_{i}$ are non-negative bounded functions for $\alpha=0)$, then

$$
\lim _{x \rightarrow \infty} \operatorname{dist}(F(x), \mathbb{P})=0
$$

where $\mathbb{P}$ is the manifold of equilibrium distributions associated with the invariants (14) of $F$. If there are only finitely many equilibrium distributions in $\mathbb{P}$, then there is an equilibrium distribution $P$ in $\mathbb{P}$, such that $\lim _{x \rightarrow \infty} F(x)=P$.

Remark 2 A key point in the proof of Theorem 1 (as well as - with x replaced with $t$ - for Theorem 2 below), cf. [17],[18], is that

$$
\int_{0}^{\infty} \frac{d}{d x} \mathcal{H}[F] d x=\lim _{x \rightarrow \infty} \mathcal{H}[F](x)-\mathcal{H}[F](0)
$$

is a finite non-positive number, since $\mathcal{H}[F]=\mathcal{H}[F](x)$ is bounded - $\mu(y)$ is non-positive and bounded below, since $\mu(0)=\mu\left(\frac{1}{\alpha}\right)=0$, while $\mu^{\prime}(y)$ is strictly increasing for $0<y<\frac{1}{\alpha}$ - and differentiable almost everywhere in $\mathbb{R}_{+}$, with $\frac{d}{d x} \mathcal{H}[F]$ non-positive. For complete arguments and more details see [17], [18].

For the spatially homogeneous system

$$
\frac{d F}{d t}=Q^{\alpha}(F), t \in \mathbb{R}_{+}
$$


we obtain similar results for the trend to equilibrium, presented in Theorem 2 below, by repeating the same arguments (see also Remark 2 above), but with a modified $\mathcal{H}$-function

$$
\mathcal{H}[F]=\mathcal{H}[F](t)=\sum_{i=1}^{N} \mu\left(F_{i}(t)\right),
$$

with $\mu$ given by the expression (12), and the moments

$$
\left\{\begin{array}{l}
\widetilde{j}_{1}=\langle\mathbf{1}, F\rangle \\
\widetilde{j}_{i+1}=\left\langle p^{i}, F\right\rangle, i=1, \ldots, d, \mathbf{1}=(1, \ldots, 1) \in \mathbb{R}^{N} . \\
\widetilde{j}_{d+2}=\left\langle|\mathbf{p}|^{2}, F\right\rangle
\end{array}\right.
$$

Then we obtain the $\mathcal{H}$-theorem

$$
\frac{d}{d t} \mathcal{H}[F]=\sum_{i=1}^{N} \frac{d F_{i}}{d t} \log \frac{F_{i}}{\Psi_{\alpha}\left(F_{i}\right)}=\left\langle\log \frac{F}{\Psi_{\alpha}(F)}, Q^{\alpha}(F)\right\rangle \leq 0 .
$$

By applying relation (10) for the system (15), one obtain that $\widetilde{j}_{1}, \ldots, \widetilde{j}_{d+2}$ are independent of $t$ in the spatially homogeneous case, and for some fixed numbers $\widetilde{j}_{1}, \ldots, \widetilde{j}_{d+2}$, we denote by $\widetilde{\mathbb{P}}$ the manifold of all equilibrium distributions $F=P$ (given by equation.(11)), with moments (16).

Theorem 2 If $F=F(t)$ is a solution to the system (15), such that $0 \leq F_{i} \leq \frac{1}{\alpha}$ ( $F_{i}$ is a non-negative bounded function if $\alpha=0$ ), then

$$
\lim _{t \rightarrow \infty} \operatorname{dist}(F(t), \widetilde{\mathbb{P}})=0
$$

where $\widetilde{\mathbb{P}}$ is the manifold of equilibrium distributions associated with the invariants (16) of $F$. If there are only finitely many equilibrium distributions in $\widetilde{\mathbb{P}}$, then there is an equilibrium distribution $P$ in $\widetilde{\mathbb{P}}$, such that $\lim _{t \rightarrow \infty} F(t)=P$.

Remark 3 Let $I_{N}=\{1, \ldots, N\}$ and $1 \leq m \leq n \leq N-m$, and denote

$$
\begin{aligned}
& Q_{i}^{\alpha}(F)=\sum_{1 \leq m \leq n \leq N-m} a_{m n} Q_{i}^{\alpha, m n}(F), a_{m n} \geq 0 \text {, } \\
& Q_{i}^{\alpha, m n}(F)=\sum_{\substack{I^{\prime}, I^{\prime \prime} \subset I_{N} \\
\left|I^{\prime}\right|=n,\left|I^{\prime \prime}\right|=m}} \Gamma_{I^{\prime}}^{I^{\prime \prime}}\left(\sum_{k \in I^{\prime}} \delta_{i k}-\sum_{k \in I^{\prime \prime}} \delta_{i k}\right)\left(\prod_{j \in I^{\prime}} F_{j} \prod_{j \in I^{\prime \prime}} \Psi_{\alpha}\left(F_{j}\right)-\prod_{j \in I^{\prime \prime}} F_{j} \prod_{j \in I^{\prime}} \Psi_{\alpha}\left(F_{j}\right)\right) \\
& =\sum_{\substack{I^{\prime}, I^{\prime \prime} \subset I \\
\left|I^{\prime}\right|=n,\left|I^{\prime}\right|=m}} \Gamma_{I^{\prime}}^{I^{\prime \prime}}\left(\sum_{k \in I^{\prime}} \delta_{i k}-\sum_{k \in I^{\prime \prime}} \delta_{i k}\right) \prod_{j \in I^{\prime} \cup I^{\prime \prime}} F_{j}\left(\prod_{j \in I^{\prime}} \frac{F_{j}}{\Psi_{\alpha}\left(F_{j}\right)}-\prod_{j \in I^{\prime \prime}} \frac{F_{j}}{\Psi_{\alpha}\left(F_{j}\right)}\right), \\
& \Psi_{\alpha}\left(F_{l}\right)=\left(1-\alpha F_{l}\right)^{\alpha}\left(1+(1-\alpha) F_{l}\right)^{1-\alpha} .
\end{aligned}
$$

where $\Gamma_{I^{\prime}}^{I^{\prime \prime}}=0$, if the relations

$$
\sum_{k \in I^{\prime}} \mathbf{p}_{k}=\sum_{k \in I^{\prime \prime}} \mathbf{p}_{k} \text { and } \sum_{k \in I^{\prime}}\left|\mathbf{p}_{k}\right|^{2}=\sum_{k \in I^{\prime \prime}}\left|\mathbf{p}_{k}\right|^{2}
$$

are not satisfied (can be changed to other collision invariants). Then, in a similar way as above, we can obtain corresponding results for the system (1). However, if at least one $a_{m n}$ such that $m \neq n$ is nonzero, then the collision invariants (for normal DVMs) will be of the form

$$
\phi=\mathbf{b} \cdot \mathbf{p}+c|\mathbf{p}|^{2},
$$

and we will have to exclude the moments $j_{1}$ and $\widetilde{j_{1}}$ from the moments $(14)$ and (16), respectively, for Theorem 1 and Theorem 2 to stay valid. A drawback is that, in general, it will not be clear how to construct the sets $\mathcal{P}$ to obtain normal DVMs. An example when such generalizations (with $\alpha=0$ ) are of interest is for excitations in a Bose gas interacting with a Bose-Einstein condensate [19, 20, 21, 22, 23, 24]. However, even if the momentum is still assumed to be conserved during a collision, the energy conserved will (in the general case) be different from the kinetic one conserved by the relations (4). Furthermore, the equation will (in the general case) also be coupled by a GrossPitaevskii equation for the density of the condensate. 


\section{LINEARIZED COLLISION OPERATOR}

For any $\alpha, 0 \leq \alpha \leq 1$, one can derive that

$$
\begin{aligned}
\Psi_{\alpha}^{\prime}(y) & =-\alpha^{2}(1-\alpha y)^{\alpha-1}(1+(1-\alpha) y)^{1-\alpha}+(1-\alpha)^{2}(1-\alpha y)^{\alpha}(1+(1-\alpha) y)^{-\alpha} \\
& =\Psi_{\alpha}(y)\left(\frac{1-2 \alpha-\alpha(1-\alpha) y}{(1-\alpha y)(1+(1-\alpha) y)}\right)=\Psi_{\alpha}(y)\left(\frac{1-2 \alpha-\alpha(1-\alpha) y}{1+y(1-2 \alpha-\alpha(1-\alpha) y)}\right),
\end{aligned}
$$

and, hence, that

$$
\frac{\Psi_{\alpha}\left(P_{i}\right)-\Psi_{\alpha}^{\prime}\left(P_{i}\right) P_{i}}{P_{i} \Psi_{\alpha}\left(P_{i}\right)}=\frac{1}{P_{i}\left(1-\alpha P_{i}\right)\left(1+(1-\alpha) P_{i}\right)} .
$$

Furthermore, if we denote

$$
F=P+R^{1 / 2} f, \text { with } R=P(1-\alpha P)(1+(1-\alpha) P) \text { and } \frac{P}{\Psi_{\alpha}(P)}=M,
$$

in the system (1), and ignore all terms of second order; we obtain

$$
\frac{\partial f_{i}}{\partial t}+\mathbf{p}_{i} \cdot \nabla_{\mathbf{x}} f_{i}+(L f)_{i}=0
$$

where $L$ is the linearized collision operator $(N \times N$ matrix $)$ given by

$$
(L f)_{i}=\sum_{j, k, l=1}^{N} \frac{\Gamma_{i j}^{k l}}{R_{i}^{1 / 2}}\left(P_{i j}^{k l} f_{i}+P_{j i}^{k l} f_{j}-P_{k l}^{i j} f_{k}-P_{l k}^{i j} f_{l}\right), i=1, \ldots, N .
$$

Note that for bosons and fermions, we obtain (cf. [12])

$$
R=P(1+P) \text { and } R=P(1-P),
$$

respectively, and for semions $(\alpha=1 / 2)$ we obtain

$$
R=P\left(1-\frac{P^{2}}{4}\right)
$$

Here we, by denoting

$$
\Pi_{i j}^{k l}(g)=g_{i} g_{j} \Psi_{\alpha}\left(g_{k}\right) \Psi_{\alpha}\left(g_{l}\right)-g_{k} g_{l} \Psi_{\alpha}\left(g_{i}\right) \Psi_{\alpha}\left(g_{j}\right),
$$

have that

$$
\begin{aligned}
P_{i j}^{k l} & =\left.\frac{\partial \Pi_{i j}^{k l}\left(P+R^{1 / 2} f\right)}{\partial f_{i}}\right|_{f=0}=R_{i}^{1 / 2}\left(P_{j} \Psi_{\alpha}\left(P_{k}\right) \Psi_{\alpha}\left(P_{l}\right)-P_{k} P_{l} \Psi_{\alpha}^{\prime}\left(P_{i}\right) \Psi_{\alpha}\left(P_{j}\right)\right) \\
& =\frac{P_{i} P_{j} \Psi_{\alpha}\left(P_{k}\right) \Psi_{\alpha}\left(P_{l}\right)}{R_{i}^{1 / 2}} \frac{\Psi_{\alpha}\left(P_{i}\right)-\Psi_{\alpha}^{\prime}\left(P_{i}\right) P_{i}}{P_{i} \Psi_{\alpha}\left(P_{i}\right)} R_{i}=\frac{P_{i} P_{j} \Psi_{\alpha}\left(P_{k}\right) \Psi_{\alpha}\left(P_{l}\right)}{R_{i}^{1 / 2}}
\end{aligned}
$$

since, by the relations (20) and (21),

$$
\frac{\Psi_{\alpha}\left(P_{i}\right)-\Psi_{\alpha}^{\prime}\left(P_{i}\right) P_{i}}{P_{i} \Psi_{\alpha}\left(P_{i}\right)} R_{i}=1
$$

By the relations (4) and (11), we obtain the relation

$$
P_{i} P_{j} \Psi_{\alpha}\left(P_{k}\right) \Psi_{\alpha}\left(P_{l}\right)=P_{k} P_{l} \Psi_{\alpha}\left(P_{i}\right) \Psi_{\alpha}\left(P_{j}\right)
$$

for $\Gamma_{i j}^{k l} \neq 0$. and hence, by relations (3), (22), (23), and (25), we obtain the equality

$$
\langle g, L f\rangle=\frac{1}{4} \sum_{i, j, k, l=1}^{N} \Gamma_{i j}^{k l} P_{i} P_{j} \Psi_{\alpha}\left(P_{k}\right) \Psi_{\alpha}\left(P_{l}\right)\left(\frac{f_{i}}{R_{i}^{1 / 2}}+\frac{f_{j}}{R_{j}^{1 / 2}}-\frac{f_{k}}{R_{k}^{1 / 2}}-\frac{f_{l}}{R_{l}^{1 / 2}}\right)\left(\frac{g_{i}}{R_{i}^{1 / 2}}+\frac{g_{j}}{R_{j}^{1 / 2}}-\frac{g_{k}}{R_{k}^{1 / 2}}-\frac{g_{l}}{R_{l}^{1 / 2}}\right) .
$$


Then it is immediate that the matrix $L$ is symmetric and positive semi-definite, i.e.

$$
\langle g, L f\rangle=\langle L g, f\rangle \text { and }\langle f, L f\rangle \geq 0,
$$

for all $g=g(\mathbf{p})$ and $f=f(\mathbf{p})$.

Furthermore, by the relation (26), $\langle f, L f\rangle=0$ if and only if

$$
\frac{f_{i}}{R_{i}^{1 / 2}}+\frac{f_{j}}{R_{j}^{1 / 2}}=\frac{f_{k}}{R_{k}^{1 / 2}}+\frac{f_{l}}{R_{l}^{1 / 2}}
$$

for all indices satisfying $\Gamma_{i j}^{k l} \neq 0$. We denote $f=R^{1 / 2} \phi$ in equality (27) and obtain the relation (8). Hence, since $L$ is semi-positive,

$$
L f=0 \text { if and only if } f=R^{1 / 2} \phi,
$$

where $\phi$ is a collision invariant (8). Hence, for normal models the null-space $N(L)$ is

$$
N(L)=\operatorname{span}\left(R^{1 / 2}, R^{1 / 2} p^{1}, \ldots, R^{1 / 2} p^{d}, R^{1 / 2}|\mathbf{p}|^{2}\right), R=P(1-\alpha P)(1+(1-\alpha) P) .
$$

Remark 4 More generally, we can consider the collision operator (19) and obtain corresponding results for the linearized collision operator $L$ by similar arguments. In particular, the linearized operator $L$ is symmetric and positive semi-definite. However, if at least one $a_{m n}$ such that $m \neq n$ is nonzero then for normal models the following holds

$$
N(L)=\operatorname{span}\left(R^{1 / 2} p^{1}, \ldots, R^{1 / 2} p^{d}, R^{1 / 2}|\mathbf{p}|^{2}\right), R=P(1-\alpha P)(1+(1-\alpha) P) .
$$

\section{Linearized Spatially Homogeneous Equation}

The Cauchy problem for linearized spatially homogeneous equation reads

$$
\frac{d f}{d t}+L f=0, f(0)=f_{0},
$$

for some $f_{0} \in \mathbb{R}^{N}$, and has a bounded solution

$$
f(t)=e^{-t L} f_{0},
$$

such that, for any orthogonal basis $\left\{y_{1}, \ldots, y_{d+2}\right\}$ of the null-space $N(L)$ of $L(28)$,

$$
f(t) \rightarrow \sum_{i=1}^{d+2} \frac{\left\langle y_{i}, f_{0}\right\rangle}{\left\langle y_{i}, y_{i}\right\rangle} y_{i} \text {, as } t \rightarrow \infty .
$$

\section{Linearized Half-Space Problems}

The linearized steady system in a slab-symmetry reads

$$
B \frac{d f}{d x}+L f=0, f(0)=f_{0}, \text { with } B=\operatorname{diag}\left(p_{1}^{1}, \ldots, p_{N}^{1}\right),
$$

for some $f_{0} \in \mathbb{R}^{N}$, where

$$
\mathbf{x}=\left(x=x^{1}, x^{2}, \ldots, x^{d}\right) \text { and } \mathbf{p}=\left(p^{1}, \ldots, p^{d}\right) .
$$

Due to the matrix $B$, here the situation will be much more intricate than for the spatially homogeneous equation. However, since above, some general properties of the "classical" discrete Boltzmann equation (obtained by letting $\Psi_{\alpha}=1$ in the collision operator (2)), have been shown also for the discrete quantum Boltzmann equation, the general results obtained for linearized half-space problems for the discrete Boltzmann equation obtained in [10, 11] (also cf. [24]) hold also for the discrete quantum Boltzmann equation presented here. We note that the numbers of positive, negative, and zero eigenvalues, respectively, of the symmetric $(d+2) \times(d+2)$ matrix $K$ with elements

$$
k_{i j}=\left\langle y_{i}, B y_{j}\right\rangle,
$$

where $\left\{y_{1}, \ldots, y_{d+2}\right\}$ is any basis of the null-space $N(L)$ of $L(28)$, is of great importance for these results (see for example $[10,11,24,12])$. 


\section{CONCLUDING REMARKS}

We have presented a general discrete velocity model (DVM) of Boltzmann equation for anyons (or Haldane statistics), and considered it in the lines of previous studies of the "classical" discrete Boltzmann equation, see e.g. [10, 11, 12]. As two limiting cases the Nordheim-Boltzmann equation for bosons and fermions are also included. The equilibrium distributions are shown to satisfy a transcendental equation (11) (a corresponding equation for the continuous case was first presented in [16]). In certain cases the equation can be analytically solved; we have presented the results in the simplest cases: for bosons, fermions, and so called semions. By a suitable choice of $\mathcal{H}$-function(s), we obtained $\mathcal{H}$-theorem(s), and, thereby could state trend to equilibrium in the spatially homogeneous, as well as, in the planar stationary case. By linearizing around an equilibrium distribution, in a suitable way, we obtained a linearized operator with similar properties to the linearized operator for the classical discrete Boltzmann operator, i.e. a symmetric, positive semi-definite operator, with a null-space of the same dimension as the vector space of the collision invariants. Then the solution of the Cauchy problem for linearized spatially homogeneous equation was immediate, while the results for the linearized steady half-space problems in a slab-symmetry are more intricate. However, while they are not presented here, the results can be found in $[10,11,24]$, where they were presented for linearized operators of other discrete Boltzmann equations, but with similar properties. Note that half-space problems for the non-linear Nordheim-Boltzmann equation (i.e. for bosons and fermions) are considered in [12]. We refer the reader to [12] for the obtained results. However, in the general case there are no such results (for the non-linear equation) yet. We also stress that all results presented here are independent of the (finite) number of velocities considered.

All results are in general not depending on which collision invariants that are assumed, so for example other conserved energies than the assumed kinetic one can be considered. However, in implementations, it might be a difficulty to find "good" velocity sets, not to have extra (spurious) collision invariants in plus to the desired (physical) collision invariants (9). On the other hand, for the collision invariants considered, there are well-known procedures to obtain DVMs without spurious collision invariants, see e.g. [13, 14, 15]. The results can also be generalized to more general collision operators (28), cf. Remarks 3 and 4, where in many cases mass will not be conserved, and therefore one might obtain similar difficulties to find "good" velocity sets as in the case of exchanging energy. On the other hand from a theoretical point of view there is nothing preventing such generalizations.

We also want to stress that the results presented here can be generalized to mixtures, and particles with a discrete number of different energy levels, with the approaches presented in [25, 26, 12, 27].

Finally, up to our knowledge and belief, corresponding results - to the ones presented here for DVMs - are also valid in the case of a continuous velocity variable (with a suitable choice of collision kernels). However, even if some of them - in a clear way - can be obtained (correspondingly) as above, some others will be more demanding to show.

\section{ACKNOWLEDGMENTS}

The author would like to thank Y. Wondmagegne and M. Vinerean Bernhoff for their comments on the manuscript.

\section{REFERENCES}

[1] J. Leinaas and J. Myrheim, Nuovo Cim. B 37, 1-23 (1977).

[2] F. Wilczek, Phys. Rev. Lett. 49, 957-959 (1982).

[3] L. W. Nordheim, Proc. Roy. Soc. London Ser. A 119, 689-698 (1928).

[4] E. A. Uehling and G. E. Uhlenbeck, Phys. Rev. 43, 552-561 (1933).

[5] F. D. Haldane, Phys. Rev. Lett. 67, 937-940 (1991).

[6] R. K. Bhaduri, R. S. Bhalerao, and M. V. N. Murthy, J. Stat. Phys. 82, 1659-1668 (1996).

[7] L. Arkeryd, J. Stat. Phys. 150, 1063-1079 (2013).

[8] L. Arkeryd and A. Nouri, SIAM J. Math. Anal. 47, 4720-4742 (2015).

[9] L. Arkeryd and A. Nouri, Kinet. Relat. Models 12, 323-346 (2019).

[10] A. V. Bobylev and N. Bernhoff, in Lecture Notes on the Discretization of the Boltzmann Equation, edited by N. Bellomo and R. Gatignol (World Scientific, 2003), pp. 203-222.

[11] N. Bernhoff, Riv. Mat. Univ. Parma 9, 73-124 (2008).

[12] N. Bernhoff, Kinet. Relat. Models 10, 925-955 (2017).

[13] A. V. Bobylev and C. Cercignani, J. Stat. Phys. 97, 677-686 (1999). 
[14] A. V. Bobylev and M. C. Vinerean, J. Stat. Phys. 132, 153-170 (2008).

[15] A. V. Bobylev, M. C. Vinerean, and A. Windfall, Kinet. Relat. Models 3, 35-58 (2010).

[16] Y.-S. Wu, Phys. Rev. Lett. 73, 922-925 (1994).

[17] C. Cercignani, R. Illner, M. Pulvirenti, and M. Shinbrot, J. Stat. Phys. 52, 885-896 (1988).

[18] N. Bernhoff and A. Bobylev, Commun. Math. Sci. 5, 815-832 (2007).

[19] T. R. Kirkpatrick and J. R. Dorfman, J. Low Temp. Phys. 58, 301-331 (1985).

[20] E. Zaremba, T. Nikuni, and A. Griffin, J. Low Temp. Phys. 116, 277-345 (1999).

[21] L. Arkeryd and A. Nouri, Comm. Math.Phys. 310, 765-788 (2012).

[22] E. Gust and L. Reichl, J. Low Temp. Phys. 170, 43-59 (2013).

[23] L. Arkeryd and A. Nouri, Kinet. Relat. Models 6, 671-686 (2013).

[24] N. Bernhoff, J. Stat. Phys. 159, 358-379 (2015).

[25] N. Bernhoff and M. C. Vinerean, J. Stat. Phys. 165, 434-453 (2016).

[26] N. Bernhoff, AIP Conference Proceedings 1786, p. 040005 (2016).

[27] N. Bernhoff, J. Stat. Phys. 172, 742-761 (2018). 